\title{
Crecimiento económico en las manufacturas mexicanas: un análisis de contabilidad del crecimiento en las entidades federativas, 1988-2003
}

\section{Economic growth in Mexican manufacturing: an analysis of growth accountancy in the federal states, 1988-2003}

\author{
Adrián de León-Arias \\ EdNA Edith PARRA-DE LA TORRE*
}

\begin{abstract}
In this paper, taking advantage of the available census information from 1988 to 2003, we explore the characteristics of the dynamic of manufacturing productivity in the Mexican states applying the growth accounting methodology. As a conclusion, we found that some geographical patterns of growth can be identified in the West and East Central and Northern Border and these are centered in states such as Mexico, Jalisco, Baja California, and Chihuahua, among other, which can be identified as the winners, while the Federal District, one of the states with the largest manufacturing employment, becomes the loser in terms of manufacturing economic growth. Regarding the sources of growth in the winner states, relevant is the contribution of labor and to a lesser extent, total factor productivity.
\end{abstract}

Keywords: Mexico, economic growth, regional economic development.

\section{Resumen}

En este artículo, tomando ventaja de la información censal disponible durante el periodo de 1988 a 2003, exploramos las características de la dinámica de la productividad manufacturera por entidad federativa, con base en la llamada contabilidad del crecimiento. Como conclusión, se encuentran algunos patrones geográficos del crecimiento identificados en el centro-occidente, centro-oriente y la frontera norte, y dicho crecimiento se centra en estados como México, Jalisco, Baja California y Chihuahua, entre otros, que pueden ser identificados como estados ganadores, mientras que el Distrito Federal, una de las entidades federativas con mayor actividad manufacturera, es la gran perdedora en términos de crecimiento económico en este rubro. En cuanto a las fuentes de crecimiento en los estados ganadores, destaca la contribución del trabajo y en menor medida, la productividad factorial total.

Palabras clave: México, crecimiento económico, desarrollo económico regional.

*Universidad de Guadalajara, México. Correos-e: Laa02511@cucea.udg.mx, leonarias@yahoo. com; ednapt@gmail.com. 


\section{Introducción}

Si bien en las últimas dos décadas ha habido una recuperación relativa del crecimiento económico en las manufacturas mexicanas, no se ha profundizado lo suficiente en la identificación de los patrones espaciales donde se ha expresado dicho crecimiento. Por tanto, es de interés para el análisis del desarrollo económico regional preguntarse si existe un patrón geográfico de crecimiento de la productividad en los años recientes en México y qué entidades federativas son las ganadoras y cuáles las perdedoras en términos de desempeño productivo, así como qué fuentes de crecimiento explican su buen o mal desempeño.

Al respecto, economistas como Livas y Krugman (1992) y Hanson (1994) han observado diferencias significativas en los patrones de crecimiento del empleo industrial entre las regiones, entre las que destaca el rápido crecimiento de la actividad manufacturera en la frontera norte y la pérdida de dicha actividad en las grandes ciudades de México. Tales economistas han argumentado que dichos cambios están claramente relacionados con el proceso de apertura comercial. Por su parte, De León $(2000,2001,2008,2009)$ ha identificado cambios en la dinámica y las fuentes de productividad en dichas regiones. Sin embargo, queda pendiente un análisis espacial definido por criterios geográficos, por estado y de más largo periodo que permita responder a las observaciones planteadas anteriormente.

Este estudio se centra en las manufacturas porque es en este sector donde se percibe más directamente el efecto de la apertura comercial, ya que concentra el mayor número de actividades relacionadas con los bienes comerciables; mientras que estas actividades fueron algunas de las áreas productivas con mayor protección comercial y, por tanto, son aquéllas que resultaron más afectadas por la apertura, además de que mostraron un desempeño productivo aceptable antes de ella. ${ }^{1}$

Así, en esta investigación, que tiene por objeto identificar los hechos estilizados del crecimiento económico en México desde una perspectiva espacial, se analizan los patrones de crecimiento económico de las manufacturas en su dimensión geográfica y por entidad federativa durante el periodo 1988-2003. En particular, en esta investigación se concluye que en dicho periodo ${ }^{2}$ existe un patrón geográfico de crecimiento de la pro-

\footnotetext{
${ }^{1}$ Para el análisis sectorial, véase Guillermo y Tanka (2007), quienes identifican grandes cambios en la manufactura para el mismo periodo de estudio y con metodología similar.

${ }^{2}$ Esta investigación abarca hasta el año 2003, fecha del último censo industrial disponible. Existe información más reciente, incluso anual, por entidad federativa, en particular sobre producto interno bruto; sin embargo, los datos provienen de encuestas u otro tipo de información que no incluye a todas las empresas manufactureras ni los datos sobre capital (activos fijos), como sí lo hacen los censos industriales o de manufacturas.
} 
ductividad centrado en diversas entidades del centro-occidente, el centrooriente y la frontera norte, entre las que destacan los estados de México, Jalisco, Baja California y Chihuahua, a los que se podría considerar como los ganadores en términos de crecimiento económico, mientras que el Distrito Federal -que para los efectos de este trabajo se considera un estado, aunque no lo sea estrictamente en términos de administración política-, entidad que presenta la mayor actividad manufacturera, es la gran perdedora en los mismos términos. Entre las fuentes de crecimiento de los estados ganadores destaca la mayor contribución del trabajo, mientras que la productividad factorial total contribuye en menor medida.

Mientras que el estudio del crecimiento económico y de la productividad en la economía mexicana ha puesto mayor énfasis en un análisis agregado o sectorial en el ámbito nacional, en esta investigación se privilegia el estudio del crecimiento económico desde la perspectiva espacial. Es relevante hacer notar que en la literatura al respecto los análisis recientes de crecimiento económico regional en México se han enfocado en el desempeño del producto per cápita, ya sea regional o por estados (Fuentes et al., 2003; Díaz-Bautista y Sáenz, 2002; Esquivel y Meesmacher, 2002), por lo que no se refieren específicamente a la productividad laboral o factorial en su totalidad, además de que se han orientado a evaluar el patrón de convergencia/divergencia regional. Por otro lado, aunque existe un gran número de estudios que han analizado la dinámica de la productividad factorial total, no ha sido con una perspectiva regional, sino más bien sectorial (De la Torre, 2000; Guillermo y Tanka, 2007). Un trabajo menos reciente es el de Hernández Laos (1984), quien estudia la productividad regional en las manufacturas mexicanas para el periodo previo a 1975. Este artículo actualiza en mucho el trabajo mencionado y aporta un análisis no realizado en la literatura al respecto, aprovechando la disponibilidad de la riqueza de información censal sobre las manufacturas estatales.

Una implicación relevante de este ejercicio es que las diferencias en la productividad es reflejo de un mejor uso de los recursos de las tecnologías existentes. Y aun si nuestra metodología da cuenta de diferencias en la productividad de los factores en el ámbito de entidad federativa, ${ }^{3}$ queda un componente del valor agregado, la PFT, propio de la acción de los productores en cada una de ellas. En ese sentido está determinado exógenamente. Aunque es cierto que esta PFT estatal estaría relacionada con características propias de los espacios geográficos, la metodología de contabilidad de crecimiento no identifica cuáles características son rele-

\footnotetext{
${ }^{3}$ De hecho, una característica de la metodología desarrollada en este estudio, es que la productividad de los factores en el ámbito estatal puede diferir mientras se obtiene a través de la participación del ingreso de cada uno de los factores en el valor agregado en el ámbito de entidad federativa.
} 
vantes. Existen análisis que han buscado avanzar en la identificación de las características mencionadas mediante el establecimiento de relaciones significativas entre rasgos propios de las regiones con PFT. En el caso de este artículo, éste sería un paso posterior, en tanto exige una amplia investigación de los determinantes de la productividad regional, aquí sólo dejamos el análisis a nivel de identificación de la PFT donde posteriores estudios habrán de aprovechar esta base de datos. ${ }^{4}$

Este artículo, aparte de la introducción, se desarrolla como sigue: en la primera sección, con el objeto de contar con un marco de análisis para este estudio, se presenta la estimación de la dinámica de la productividad factorial total de la manufactura mexicana para el periodo 1988-2003, con una descripción de la metodología utilizada y una comparación con el periodo previo, de 1970 a 1988. En la segunda sección se describen, como parte central de este trabajo, la dinámica de la productividad factorial total y las fuentes de crecimiento en la manufactura por estados para el periodo de análisis, así como la identificación de las manufacturas estatales ganadoras y perdedoras, y sus fuentes de crecimiento. En la última parte se presentan las conclusiones.

Cabe señalar que en este artículo, puesto que se utilizan como fuente los censos económicos, se explota la única base de información que, aun con limitaciones de diversos tipos, que se comentan más adelante, ofrece la mayor cobertura.

\section{Dinámica de la productividad factorial total de la manufactura mexicana: metodología y resultados nacionales, 1970-1988 y 1988-2003}

Con el objeto de analizar el crecimiento económico en el contexto de la productividad factorial total ( $\mathrm{PFT}$ ) para las manufacturas mexicanas, en este apartado primero se presenta la metodología aplicada en este trabajo para su cálculo, y a continuación se estima dicha productividad. En particular, se hacen estimaciones de la PFT para los años 1970-1988 y 19882003, así como para los periodos 1988-1998 y 1998-2003. Con ello se intenta dar cuenta del desempeño reciente y de las dos fases que podrían ser relevantes para el análisis; el periodo 1988-1998 cubre la consolidación del proceso de apertura comercial y el periodo 1998-2003, los últimos años con información disponible, después de dicha consolidación.

En este artículo se adopta la metodología convencional de contabilidad del crecimiento desarrollada por Solow (1957) y ampliada por Denison

${ }^{4}$ En tal sentido, en este artículo se incluyen en un anexo los datos pertinentes a cada una de las entidades federativas y para cada uno de los periodos de análisis. 
(1962), ${ }^{5}$ que consiste en identificar el cambio en la PFT como un residual del crecimiento del producto una vez descontado el cambio en el uso de los factores, ponderados por la participación de sus remuneraciones en el total del valor agregado. De esta manera, la PFT se define como el cambio en el producto que no puede ser atribuido a un cambio en el uso de los factores, y que refleja un conjunto de elementos que definen el hacer más con menos, característica básica del crecimiento económico. Aunque este método tiene múltiples restricciones, lo hacen particularmente atractivo su amplio uso como medida convencional de productividad, la facilidad de su cálculo y particularmente relevante para análisis comparativos. De hecho, incluso en un componente básico de los análisis del crecimiento económico, para una muestra de aplicaciones recientes de esta metodología, véase el número de invierno de 2008 del Journal of Economic Perspectives, donde se utiliza para analizar temas de crecimiento económico reciente, así como Cornberg y Pérez García (2010), quienes analizan las fuentes del crecimiento y productividad en Europa y América Latina.

En esta metodología se parte del supuesto de que el producto (valor agregado censal) manufacturero es una función del capital (valor de los activos fijos brutos), trabajo (número de trabajadores) y tiempo, que se combinan por medio de un conjunto de tecnologías de producción. Así, las condiciones necesarias para la optimización en equilibrio de la economía a través de un agente representativo, suponiendo rendimientos constantes a escala y competencia perfecta en el mercado de los factores, implican que las elasticidades del producto respecto del capital y el trabajo son iguales a la participación de los rendimientos de los factores en el costo total. Por tanto, las participaciones del capital y el trabajo en relación con el costo total suman la unidad. En este sentido, la tasa de crecimiento del producto se expresa como la suma de las tasas de crecimiento del capital y el trabajo, cada uno ponderado por su participación en el valor agregado total, y su productividad factorial total.

En este artículo, por tanto, siguiendo la metodología convencional, el cálculo de la tasa de crecimiento de la PFT (gPFT) o (Â) se obtiene a partir de la siguiente ecuación:

$$
\mathrm{gPFT}=\hat{\mathrm{A}}=\mathrm{gQ}-[\alpha \mathrm{gK}+(1-\alpha) \mathrm{gL}]
$$

Donde gQ es el crecimiento del producto, gK el crecimiento del capital, gL el crecimiento del empleo y $\alpha$ la participación de las remuneraciones al

\footnotetext{
5 Para una muestra de aplicaciones recientes de esta metodología véase Journal of Economic Perspectives, en su número correspondiente al invierno de 2008.
} 
capital en el producto; por tanto, como se explicó anteriormente, $(1-\alpha)$ representa la participación de las remuneraciones al trabajo en el producto.

Respecto de la interpretación del cambio en la PFT, aquí se coincide con la propuesta de Jorgenson y Zvi (1967), para quienes la gPFT, es decir $\hat{A}$, se puede asociar a desplazamientos en la función de producción. Más recientemente, Harberger (1998) propone observar la PFT como una reducción en los costos reales (RCR). Esto es, la PFT también se puede observar como un resultado de hacer más con menos, mientras que una mayor tasa de PFT implica que se genera más producto con la misma cantidad de recursos, ajustados por su precio relativo (productividad). ¿Y de dónde puede provenir ese mayor producto? Harberger señala que si no es producto de rendimientos de escala, debe ser resultado de una reducción de costos, o de un uso más eficiente de los recursos. Esta interpretación tiene la ventaja de que mejora la intuición del gerente de la empresa sobre las fuentes de productividad. Para una ampliación de esta idea, véase Harberger (1998, 2005).

A fin de estimar las fuentes de crecimiento, en este artículo se parte de los datos de los censos manufactureros de 1970, 1988, 1998 y 2003. En particular, hemos utilizado el total del valor agregado censal bruto como los datos referentes al producto, el total de los activos fijos netos como los datos para capital ${ }^{6}$ y el total del empleo promedio anual como el empleo para el año en cuestión. ${ }^{7}$ La información referente al producto y el capital se presenta en términos nominales y se deflactó con el índice nacional de precios implícitos del PIB ${ }^{8}$ con base 1993.

En esta metodología las participaciones del trabajo y el capital se calculan a partir de los datos de las remuneraciones salariales totales y del valor agregado censal bruto. Es posible estimarlos con procedimientos econométricos, como en Iregui et al. (2006), pero en el caso de las manufacturas mexicanas no existe información con la periodicidad requerida.

Respecto a los coeficientes que permiten ponderar las contribuciones de capital y trabajo, sus estimaciones para los diferentes periodos que se

\footnotetext{
${ }^{6}$ Respecto de los datos de capital, siguiendo a Barro y Sala-i-Martin (1995: 348), se reconoce que lo ideal sería contar con el flujo de servicios del capital físico, pero ya que este dato no está disponible, el procedimiento típico es calcular la cantidad de capital físico de un tipo particular, en este caso activos fijos netos, y se supone que el flujo de esos servicios es proporcional a los acervos. Se han hecho algunos intentos de identificar el total de los acervos con aquellos efectivamente utilizados, esto es, el uso de la capacidad instalada. En las estimaciones que se presentan aquí no se analiza tal posibilidad por la dificultad de realizarla con la información disponible.

7 En los datos de empleo es más preciso utilizar los datos por hora-hombre, pero por carecer de los mismos se presentan los datos de empleo con base en el número de hombres ocupados. Lo que implica también que todos los trabajadores laboran en promedio jornadas similares.

${ }^{8}$ El deflactor implícito del PIB de México se genera con el sistema de cuentas nacionales y se dispone de dicho indicador para todo el periodo. Para construir la serie se utilizó el correspondiente con base en 1980, y posteriormente con base en 1993. Cabe hacer notar que se hicieron cálculos utilizando el deflactor implícito de precios manufacturados y los resultados son similares.
} 
consideran se presentan en el cuadro 1. Es importante hacer notar que mientras que para la mayoría de los países el coeficiente técnico para el capital es de 0.3 a 0.4 , para la manufactura mexicana se ubica entre 0.36 y 0.64, ambos datos se corroboran de alguna manera con los estimados para toda la economía de México por Barro y Sala-i-Martin (1995: 380381). ${ }^{9}$ Una oportunidad para ampliar esta metodología es considerar la contribución al crecimiento de la productividad del trabajo, tanto calificado como no calificado, esto es, que incorpore o no capital humano. En general, la inclusión de esta forma de capital en el trabajo se ha contabilizado en la metodología de las fuentes de crecimiento, siguiendo el trabajo pionero de Denison (1962) a partir de una amplia desagregación de categorías laborales. Desde hace dos décadas, el papel del capital humano en la productividad laboral se ha enfatizado en modelos de crecimiento económico a partir de Lucas (1988) y Mankiw et al. (1992), aunque en ambos modelos el capital humano se mide más bien a partir de las tasas de escolaridad. ${ }^{10}$ Más recientemente, Harberger (1998) ha sugerido una metodología para calcular la contribución del trabajo $[(1-\alpha) \mathrm{gL})]$ al crecimiento del producto $(\mathrm{gQ})$ a partir de identificar en el salario un componente atribuido al salario para el trabajo simple o sin calificación, como $\mathrm{w}^{*} \mathrm{y}$ a la diferencia entre el salario total promedio $\bar{w}$ y este salario $\mathrm{w}^{*}$ atribuible a capital humano, cualquiera que sea su forma, esto es: escolaridad, experiencia, habilidades, etc. Como afirman Guillermo y Tanka (2007), es relevante señalar que la separación de las fuentes de ingreso laboral $(\bar{w})$ y $\left(w^{*}\right)$ evita el problema de contabilizar la contribución de la calificación del trabajo al incremento en el producto como parte de la PFT.

Cabe señalar que la ampliación de la metodología descrita se aplicó para las manufacturas mexicanas en su conjunto en Guillermo y Tanka (2007) y para algunas regiones seleccionadas en De León (2009); sin embargo, en su aplicación en el ámbito de entidades federativas, dado el decrecimiento del empleo en algunas de ellas, para los últimos años, generan la aparición de contribuciones negativas tanto del empleo calificado como del no calificado, lo que dificulta la interpretación. Por lo anterior, se ha dejado para futuras investigaciones la ampliación de la metodología en el sentido expuesto.

En cuanto a los resultados de la productividad factorial total (PFT) en el ámbito nacional, siguiendo la metodología descrita en la ecuación (1),

\footnotetext{
${ }^{9}$ Los coeficientes de participación se estimaron mediante el promedio aritmético de la tasa de participación de las remuneraciones, el trabajo y el capital respecto al producto en el ańo inicial y el año final.

${ }^{10}$ Este requerimiento, en el caso de este artículo, implica disponer del grado de escolaridad de los trabajadores de manufactura. Véase, por ejemplo, Canudas (2001), quien evalúa la influencia del capital humano en la productividad manufacturera de México, pero que al utilizar diferentes bases de datos, limita sus conclusiones.
} 
Cuadro 1

Coeficientes de participación de capital y trabajo por entidad federativa, 1970-1988 y 1988-2003

\begin{tabular}{lcccc}
\hline & \multicolumn{2}{c}{$1970-1988$} & \multicolumn{2}{c}{$1988-2003$} \\
\multicolumn{1}{c}{ Entidad federativa } & Trabajo & Capital & Trabajo & Capital \\
\hline Aguascalientes & 0.41 & 0.59 & 0.37 & 0.63 \\
Baja California & 0.44 & 0.56 & 0.49 & 0.51 \\
Baja California Sur & 0.38 & 0.62 & 0.42 & 0.58 \\
Campeche & 0.41 & 0.59 & 0.48 & 0.52 \\
Coahuila & 0.29 & 0.71 & 0.26 & 0.74 \\
Colima & 0.41 & 0.59 & 0.29 & 0.71 \\
Chiapas & 0.31 & 0.69 & 0.19 & 0.81 \\
Chihuahua & 0.42 & 0.58 & 0.45 & 0.55 \\
Distrito Federal & 0.36 & 0.64 & 0.40 & 0.60 \\
Durango & 0.38 & 0.62 & 0.37 & 0.63 \\
Guanajuato & 0.30 & 0.70 & 0.22 & 0.78 \\
Guerrero & 0.34 & 0.66 & 0.25 & 0.75 \\
Hidalgo & 0.39 & 0.61 & 0.26 & 0.74 \\
Jalisco & 0.35 & 0.65 & 0.30 & 0.70 \\
Estado de México & 0.34 & 0.66 & 0.29 & 0.71 \\
Michoacán & 0.44 & 0.56 & 0.31 & 0.69 \\
Morelos & 0.31 & 0.69 & 0.16 & 0.84 \\
Nayarit & 0.27 & 0.73 & 0.35 & 0.65 \\
Nuevo León & 0.33 & 0.67 & 0.30 & 0.70 \\
Oaxaca & 0.31 & 0.69 & 0.14 & 0.86 \\
Puebla & 0.39 & 0.61 & 0.31 & 0.69 \\
Querétaro & 0.34 & 0.66 & 0.31 & 0.69 \\
Quintana Roo & 0.42 & 0.58 & 0.39 & 0.61 \\
San Luis Potosí & 0.30 & 0.70 & 0.26 & 0.74 \\
Sinaloa & 0.38 & 0.62 & 0.38 & 0.62 \\
Sonora & 0.35 & 0.65 & 0.35 & 0.65 \\
Tabasco & 0.37 & 0.63 & 0.19 & 0.81 \\
Tamaulipas & 0.46 & 0.54 & 0.45 & 0.55 \\
Tlaxcala & 0.38 & 0.62 & 0.30 & 0.70 \\
Veracruz & 0.41 & 0.59 & 0.33 & 0.67 \\
Yucatán & 0.39 & 0.61 & 0.32 & 0.68 \\
Zacatecas & 0.26 & 0.74 & 0.24 & 0.76 \\
Total nacional & 0.35 & 0.65 & 0.31 & 0.69 \\
\hline
\end{tabular}

Fuente: Cálculos propios con base en información de los censos industriales, de manufacturas o económicos de México (INEGI), según sea el caso. 


\section{Cuadro 2 \\ Análisis de fuentes de crecimiento, 1970-2003 (tasas de crecimiento promedio anual)}

\begin{tabular}{lcccc}
\hline & $\begin{array}{c}\text { Crecimiento del } \\
\text { producto }\end{array}$ & $\begin{array}{c}\text { Contribución del } \\
\text { capital }\end{array}$ & $\begin{array}{c}\text { Contribución del } \\
\text { trabajo }\end{array}$ & $\begin{array}{c}\text { Crecimiento de la } \\
\text { PFT }\end{array}$ \\
\hline $1970-1988$ & 3.85 & 4.38 & 1.11 & -1.64 \\
$1988-2003$ & 1.66 & -0.66 & 0.97 & 1.35 \\
$1988-1998$ & 2.24 & -0.40 & 1.73 & 0.91 \\
$1998-2003$ & 0.52 & -1.13 & -0.37 & 2.02 \\
\hline
\end{tabular}

Fuente: Cálculos propios con base en información de los censos industriales y económicos consignados en la bibliografía.

éstos se presentan en el cuadro 2 y muestran tasas de crecimiento promedio de $1.35 \%$ anual durante el periodo 1988-2003. Si se observa por subperiodos la tasa de crecimiento de la PFT, alcanza un valor de 0.91 de 1988 a 1998 y una tasa de 2.02 de 1998 a 2003. Aunque es importante hacer notar que ese crecimiento significativo en la tasa de PFT se obtiene en parte con una contribución negativa por el capital y el empleo.

Si se comparan estas tasas en un plazo más largo, también en el cuadro 2 se muestra que tales valores son significativamente mayores que los observados para el periodo 1970-1988, que mostró una tasa negativa promedio anual de $1.64 \%$, aunque con una contribución significativa tanto de empleo como de capital.

Desde luego, hay que tomar en cuenta que tales estimaciones tienen límites que provienen de la información generada en el censo con diferente calidad y cobertura a lo largo de los periodos evaluados, así como limitaciones en la construcción de los índices utilizados para la deflactación, por no contar con índices de precios encadenados. Sin embargo, las tendencias son similares a los resultados de otras estimaciones hechas para diferentes periodos con distintas fuentes de información, como la que muestran Guillermo y Tanka (2007). Aunque conviene señalar que las estimaciones de este artículo podrían ser algo menores respecto a otras hechas a partir de la encuesta industrial, como De la Torre (2000), ya que éstas incluyen información sólo de empresas grandes y medianas, mientras que los censos económicos incluyen un universo más amplio de empresas.

Aquí no entramos en el estudio de tales resultados porque no es el objetivo de este artículo. Se presentan sólo para tener un contexto del análisis más particular sobre la dinámica productiva en las manufacturas de los estados. 


\section{La dinámica de la productividad factorial total en las manufacturas estatales mexicanas}

En esta sección, con el fin de analizar el comportamiento reciente del crecimiento económico desde una perspectiva geográfica, se presenta el análisis de la productividad factorial total para las manufacturas estatales, adaptando la metodología descrita en la segunda sección. Así, a partir de la descripción de los resultados, se identifican las manufacturas estatales ganadoras y perdedoras, su patrón geográfico y las fuentes de crecimiento que explican dicho desempeño.

Respecto a la metodología, es relevante mencionar las ampliaciones hechas para adaptarla a la información estatal disponible ${ }^{11}$ en los censos económicos. Se parte de considerar que los factores productivos se combinan mediante una función de producción representativa de las manufacturas de cada entidad federativa. Por tanto, suponiendo rendimientos constantes a escala de las manufacturas estatales, así como competencia perfecta en el mercado de los factores, implican que las elasticidades del producto respecto al capital y el trabajo son iguales a las participaciones de beneficios y la depreciación -por parte del capital-y salarios -por parte del trabajo- respecto al valor agregado total. En este sentido, es completamente aplicable la metodología expuesta en la segunda sección de este artículo. Asimismo, en el cuadro 1 se presentaron las estimaciones de participación de trabajo y capital para las manufacturas estatales en los periodos seleccionados, para los cuales se puede observar que éstos se encontraban en promedio en 0.35 y $0.69 \%$, respectivamente.

Aspectos adicionales por considerar tienen que ver con que no se dispone de índices de precios en el ámbito regional, por lo que se aplicó el deflactor nacional implícito del PIв para los valores nominales del total de cada estado, con la ventaja adicional de que este procedimiento facilita la congruencia de las estimaciones del total agregado con respecto a las estimaciones estatales.

En la literatura al respecto hay estudios similares que analizan la dinámica de la productividad estatal, como el de Liu y Yoon (2000) sobre la reforma económica de China y los diferenciales de productividad regional estimados para el periodo 1986-1991, a partir de un modelo de función de producción basado en datos de panel y que muestran convergencia en el periodo de reforma. Otros estudios investigan los determinantes regionales de la PFT: para las regiones italianas, Ascari y Di Cosmo

${ }^{11}$ Similar ampliación se aplica para las entidades de Estados Unidos por Moomaw y Williams (1991). 
(2004); para la India, Kumar (2004); para Colombia, Iregui et al. (2006), y para el Reino Unido, Boddy et al. (2005).

Una vez más, aun cuando se reconoce que este método tiene múltiples restricciones, su amplio uso como medida convencional de productividad y su facilidad de cálculo lo hacen particularmente atractivo, como ya se refirió.

En cuanto a los resultados, con el objeto de contextualizar los correspondientes al periodo en estudio, se presentan los referentes a los años 1970-1988, por ser el periodo previo y porque ilustran sobre las características del patrón de crecimiento de la productividad en una economía menos liberalizada en lo comercial y una industrialización basada en la sustitución de importaciones.

Para ilustrar la presentación de los resultados, se adaptan de Harberger (1998) los diagramas sunset/sunrise, que permiten observar gráficamente la distribución del crecimiento de la PFT en los estados e identificar sus aportaciones individuales, tanto positivas como negativas, en relación con la tasa de crecimiento agregada para un periodo en particular.

Puesto que es relativamente escasa la familiaridad con los diagramas sunset/sunrise, a continuación se describe de manera detallada la forma en que se construyen. En particular, la información requerida para el diseño del diagrama se presenta en el cuadro A.1 del Apéndice, en la que se muestran los datos del periodo 1970-1988. En la columna в de dicha tabla se presentan las tasas de crecimiento de la PFT por estados, ordenadas de mayor a menor, y también se observa que estas tasas presentan valores positivos y negativos, lo cual refleja un complejo proceso de crecimiento de la productividad, con amplias variaciones y no meramente lineal, como sugiere Haberger (1998). A continuación, en la columna B, se incluye la participación del valor agregado de cada estado respecto al total nacional. En la columna D aparece el producto de multiplicar los elementos de la columna в por los de la c.

Este producto asigna a cada entidad federativa la magnitud proporcional en que contribuye al crecimiento de la productividad. De esta manera se obtiene un valor que representa su contribución relativa al total del crecimiento nacional; las contribuciones pueden ser positivas o negativas, según el valor de la tasas de crecimiento. Por tanto, las tasas de crecimiento agregadas se pueden ver como valores ponderados por la participación del estado en el total del país. Puesto que la columna в muestra las tasas de crecimiento de la PFT por estado ordenadas de mayor a menor, aparecen primero los estados que contribuyen positivamente a la productividad y los que lo hacen negativamente van al final, con su contribución ponderada por la participación de su valor agregado. Así, un estado con mayor participación obtiene más ponderación para su tasa 
de productividad. Esto tiene relación con el concepto de Harberger de ver la PFT como ahorro de costos; un estado con elevada participación y alta productividad contribuye más a disminuir los costos en el ámbito nacional; por el contrario, un estado con tasa de crecimiento negativa produce aumentando los costos. ¿En qué magnitud? Según su participación en el valor agregado.

A partir de los referentes anteriores, en la columna e se muestra la participación acumulada del valor agregado de cada uno de los estados, y en la columna F, el acumulado del producto resultante de la participación de cada entidad con su tasa de crecimiento, esto es, el acumulado de la columna D. De esta manera, es posible observar la contribución estatal a la productividad agregada conforme se va acumulando el valor agregado. Por el orden asignado, los estados con mayores tasas de crecimiento van primero y los que presentan tasas menores o negativas aparecen al final. El diagrama sunset/sunrise es la gráfica generada a partir de las dos últimas columnas del cuadro, esto es, E y F. La columna E en el eje horizontal y la columna $\mathrm{F}$ en el eje vertical. El resultado es una gráfica acumulativa a manera de u invertida, que pondera a lo largo de su expansión las contribuciones al crecimiento positivas con las negativas. Según el peso relativo de las magnitudes positivas y negativas, la $\mathrm{U}$ invertida puede tomar diferente simetrías, y cada una de ellas ilustra diferentes patrones de crecimiento. Para una ampliación de esta idea, véanse Harberger (1998, 2005) y Guillermo y Tanka (2007).

Una vez construido el primer diagrama a partir del cuadro A.1, que se presenta en la gráfica I, se analizan sus características. En primer lugar, es posible observar que durante el periodo 1970-1988 el crecimiento promedio anual agregado de la PFT (incluyendo los 32 estados) fue de menos $0.70 \%$, la última línea de la columna F. Como se explicó, la magnitud de la participación en el total del valor agregado y la distribución de las tasas de crecimiento de la PFT de cada estado determinan la forma del diagrama sunset/sunrise presentado en la gráfica I. Esta gráfica, que muestra una pendiente en ascenso, es resultado de las contribuciones acumuladas por los estados con tasas de crecimiento de la PFT positivas, mientras que la parte de la curva con pendiente decreciente es el resultado acumulado de las contribuciones de las entidades con tasas de crecimiento negativas ( 21 en este caso). En el cuadro A.1, donde se presentan la información y los cálculos para elaborar esta gráfica, en la columna $\mathrm{F}$ se observa que la tasa de crecimiento máxima de la PFT para el periodo en cuestión es del $0.12 \%$. Ésta es la tasa de crecimiento de la PFT que se podría haber alcanzado si no se hubieran tomado en consideración los estados con tasas de crecimiento negativas en el análisis nacional. Es interesante resaltar que los estados que muestran tasas de crecimiento po- 


\section{Gráfica I}

\section{Diagrama sunset/sunrise de la PFT por entidad federativa, 1970-1988}

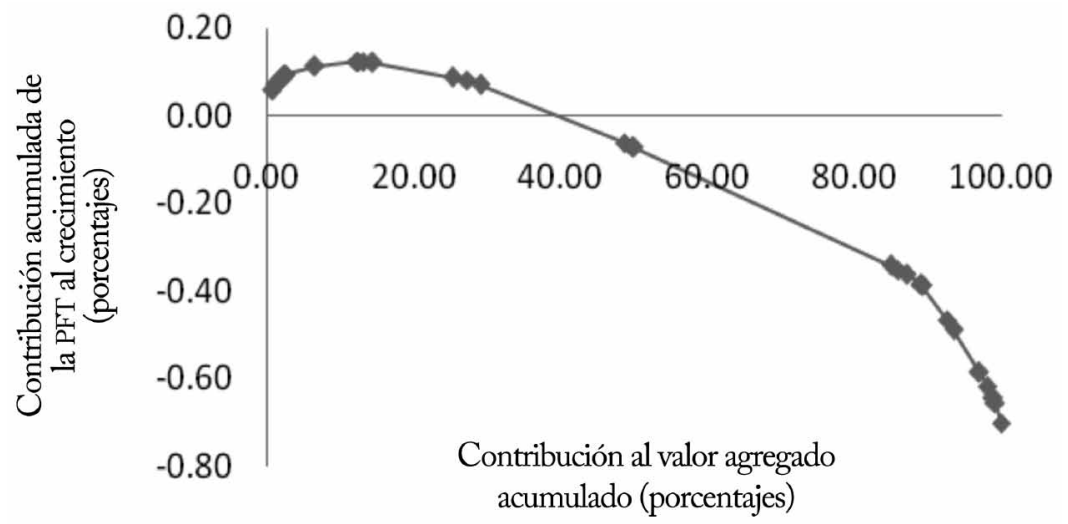

Fuente: Elaboración propia con base en información de los censos industriales, de manufacturas o económicos de México (INEGI), según sea el caso.

sitivo para la PFT sólo representan $12.47 \%$ del valor agregado acumulado en el ámbito nacional. Se puede observar que los tres estados ganadores, es decir, que presentan una disminución significativa en sus costos reales, si se sigue la interpretación de Harberger (1998) mencionada, para el periodo en análisis fueron Morelos, Oaxaca y Yucatán, mientras que los estados con un aumento significativo en sus costos reales son: Veracruz, Sonora, Durango, Aguascalientes y Michoacán.

Para el periodo 1988-2003, en la gráfica II, construida a partir de la información del cuadro A.2 del Apéndice, se observa que la tasa de crecimiento promedio anual agregada de la PFT es de $0.60 \%$; sin embargo, la tasa de crecimiento máxima que se alcanzó en el periodo es de $1.19 \%$ (en promedio anual), esto es, si se descuenta del análisis a los estados que presentan tasas de crecimiento negativas para el periodo. Es importante señalar que 25 de los 32 estados tuvieron un avance en su PFT, es decir, una reducción en sus costos reales. Los estados que presentaron mayor incremento en las tasas de crecimiento de su PFT son: Tabasco, Michoacán y Chiapas, mientras que los grandes perdedores de este periodo son el Distrito Federal, Guerrero y Nayarit.

Con la finalidad de observar con mayor precisión la dinámica de las tasas de crecimiento de la PFT en los estados, se tomó la decisión, como se mencionó, de dividir el periodo anterior en dos subperiodos: 19881998 y $1998-2003$. 


\section{Gráfica II \\ Diagrama sunset/sunrise de la PFT por entidad federativa, 1988-2003}

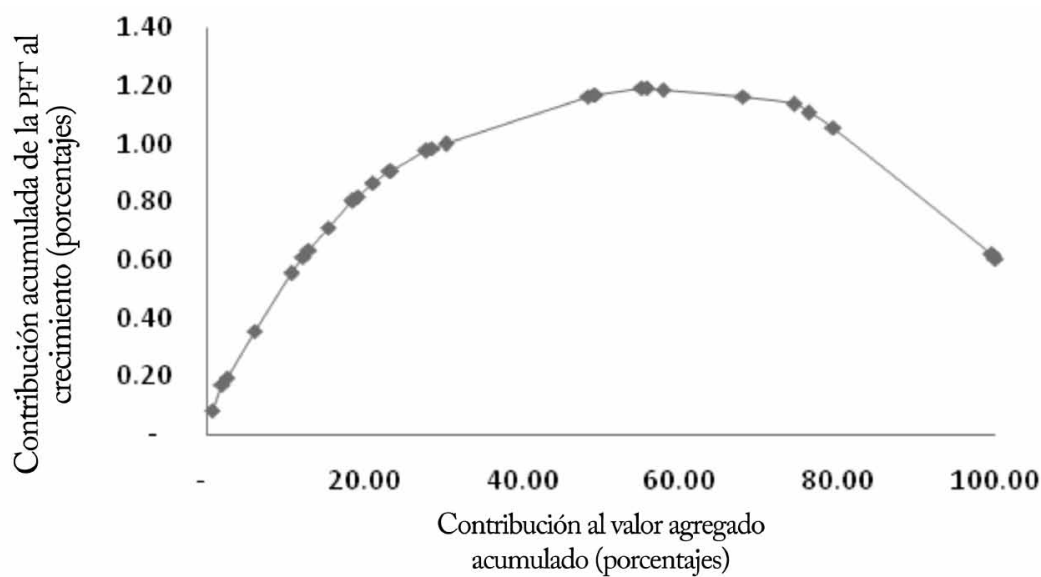

Fuente: Elaboración propia con base en información de los censos industriales, de manufacturas o económicos de México (INEGI), según sea el caso.

Así, para 1988-1998 se puede observar, a partir del cuadro A.3, que 13 entidades presentan tasas de crecimiento de la PFT negativas, por lo que la tasa de crecimiento promedio anual acumulada para el periodo es de menos de $0.15 \%$; si se realiza el análisis con los 20 estados que presentan tasas de crecimiento positivas, se observa una tasa de crecimiento máxima de la PFT de $1.33 \%$. En este subperiodo los estados que obtuvieron mayor incremento en la tasa de PFT fueron: Michoacán, Tabasco, Sonora, Querétaro, Veracruz y Guanajuato, mientras que los grandes perdedores fueron Nayarit, Oaxaca, Campeche, Hidalgo, Chiapas, Guerrero, Distrito Federal y Morelos. El diagrama sunset/sunrise correspondiente se presenta en la gráfica III.

En el periodo 1998-2003 se reduce a nueve la cantidad de estados con tasas de crecimiento negativas, entre los cuales destacan Coahuila, Nayarit, Guerrero, Distrito Federal, Sonora, Durango y Querétaro. Para el periodo de análisis se obtiene una tasa de crecimiento promedio anual de la PFT de $1.63 \%$. Sin embargo, si en el análisis no se toma en cuenta a los estados con tasas de crecimiento negativas, se obtendría una tasa máxima de $2.58 \%$. Esto significa que, a diferencia del subperiodo anterior, casi todos los estados tuvieron un incremento de la PFT, es decir, una disminución del costo real. Las entidades con mayores tasas de crecimiento de la PFT son: Chiapas, Oaxaca, Morelos, Tabasco y Quintana Roo. Es posible observar en estos dos subperiodos que estados, como Chiapas, en uno pueden ser perdedores y al siguiente estar entre los ganadores (cuadro A.4, gráfica IV). 


\section{Gráfica III}

Diagrama sunset/sunrise de la PFT por entidad federativa, 1988-1998

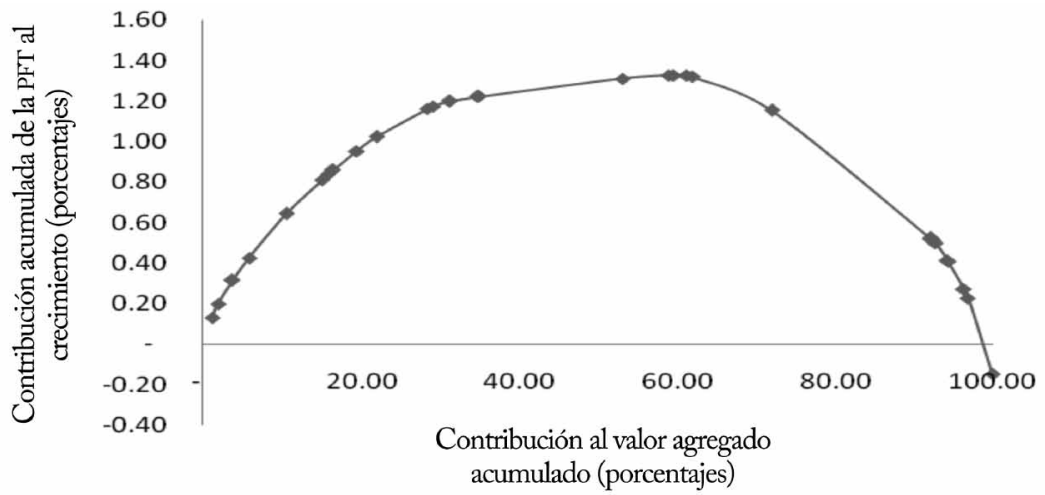

Fuente: Elaboración propia con base en información de los censos industriales, de manufacturas o económicos de México (INEGI), según sea el caso.

\section{Gráfica IV}

Diagrama sunset/sunrise de la PFT por entidad federativa, 1998-2003

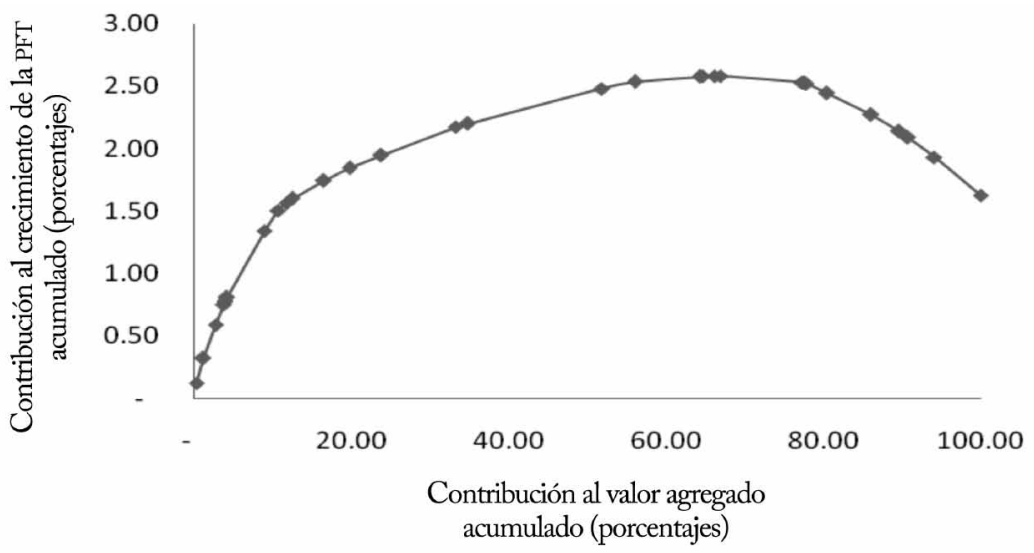

Fuente: Elaboración propia con base en información de los censos industriales, de manufacturas o económicos de México (INEGI), según sea el caso. 
Si se analiza todo el periodo en estudio, 1970-2003, como se muestra en el cuadro A.5, la industria manufacturera nacional tiene una tasa de crecimiento de la PFT de menos $0.43 \%$, pues casi todos los estados tuvieron un incremento en sus costos reales. En particular, 19 estados contribuyeron negativamente al crecimiento de la PFT y representan $83.82 \%$ de la participación en el valor agregado nacional. Si se excluye del análisis nacional sólo al Distrito Federal cambian todas las estimaciones porque éste participa con 34.99\% al valor agregado nacional, y como presenta una tasa negativa de la PFT, esto reduce considerablemente la tasa de crecimiento acumulada; si se excluye y se generan nuevamente las estimaciones, la tasa de crecimiento acumulada de la PFT se vuelve positiva, de $0.06 \%$, aunque ciertamente no es muy diferente de cero.

Del análisis anterior se puede concluir que reducir los costos reales en los subperiodos 1988-1998 y 1998-2003 representa un gran reto, lo cual se demuestra con el comportamiento de la tasa de crecimiento de PFT en los diferentes estados. En los cuadros A.3 y A.4 se observa que sólo 13 de los 32 estados tienen tasas de crecimiento de la PFT positivas y consecutivas en los dos subperiodos de análisis.

Con la finalidad de observar qué estados tuvieron buen desempeño y cuáles no en el periodo evaluado, se decidió agruparlos, de acuerdo con la dinámica de crecimiento de su PFT, como sigue: a los que presenten tasas de crecimiento positivas en la PFT en los dos subperiodos se les define como ganadores y son: Tabasco, Michoacán, Chihuahua, Veracruz, Tlaxcala, Tamaulipas, Puebla, Aguascalientes, Baja California, Estado de México, Jalisco, Zacatecas y Sinaloa, ordenados de acuerdo con sus tasas de crecimiento de la PFT, y a los que presentan tasas de crecimiento negativas en los dos subperiodos se les denomina perdedores; sin embargo, este último criterio se modificó porque sólo uno de los estados era representativo en su contribución al valor agregado nacional, por lo que sólo al Distrito Federal se le considera perdedor. Esta clasificación se presenta en el mapa I.

Como se ve en dicho mapa, si bien no existe un patrón muy claro acerca de la agrupación geográfica de las entidades federativas ganadoras, sí se observa que en el norte del país éstas son: Baja California, Chihuahua y Tamaulipas. En el centro-occidente se muestra una aglomeración de estados ganadores conformada por Jalisco, Michoacán, Zacatecas, Aguascalientes y Estado de México, mientras que en el centro-oriente se observan Veracruz, Tabasco, Tlaxcala y Puebla. En el mismo mapa se ve que el Distrito Federal, el gran perdedor del periodo de análisis, se encuentra justo en medio de las dos aglomeraciones del centro del país. Geográficamente, el estado de Sinaloa no se ubica en ninguna de las regiones definidas. 


\section{Mapa I \\ Estados ganadores y perdedores}



Fuente: Elaboración propia.

Con la finalidad de ampliar el análisis de la dinámica de crecimiento por grupos de estados, se decidió estimar la PFT agrupando las entidades en perdedoras, ganadoras y el resto de los estados para el periodo 19882003. Los resultados muestran que los ganadores presentan un patrón de acumulación de la PFT que alcanza una tasa de crecimiento máxima de 2.18\%; el Distrito Federal aparece como un gran perdedor con una tasa de crecimiento de menos $2.17 \%$, mientras que el resto del país presenta tasas de crecimiento tanto positivas como negativas y llega a tener una tasa de crecimiento máxima acumulada de $0.68 \%$; sin embargo, al incluir los estados de San Luis Potosí, Nuevo León, Coahuila, Hidalgo, Morelos, Guerrero y Nayarit, que presentan tasas negativas, se observa una tasa agregada de 0.26 por ciento.

Con el objetivo de analizar con más detalle la dinámica de las tasas de crecimiento de la PFT, se decidió continuar con el estudio en dos subperiodos: 1988-1998 y 1998-2003. En las gráficas v y vi se observa el comportamiento de las tasas de crecimiento de la PFT para cada una de las agrupaciones del periodo 1988-1998, los estados denominados ganadores presentan una tasa máxima acumulada de la PFT de $1.71 \%$. Se observa que el Distrito Federal vuelve a ser el gran perdedor del periodo ya que presenta una tasa de crecimiento de menos 3.15\%, mientras que el resto del país tiene una tasa máxima acumulada de $1.6 \%$ sólo con los estados que presentan tasas positivas, que son: Sonora, Querétaro, Guanajuato, Durango, Coahuila, San Luis Potosí y Baja California Sur; pero 


\section{Gráfica V}

Diagrama sunset/sunrise de la PFT por grupos: ganadores, perdedores y resto, 1988-1998

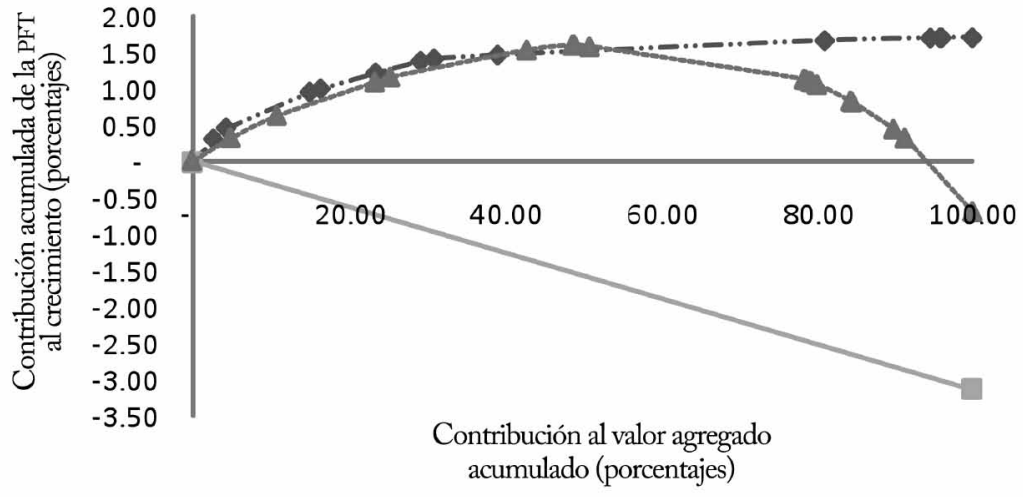

$-\uparrow$ Ganadores $\longrightarrow$ Distrito Federal $-\rightarrow-$ Resto

Fuente: Elaboración propia con base en información de los censos industriales, de manufacturas o económicos de México (INEGI), según sea el caso.

\section{Gráfica VI}

Diagrama sunset/sunrise de la PFT por grupos: ganadores, perdedores y resto, 1998-2003

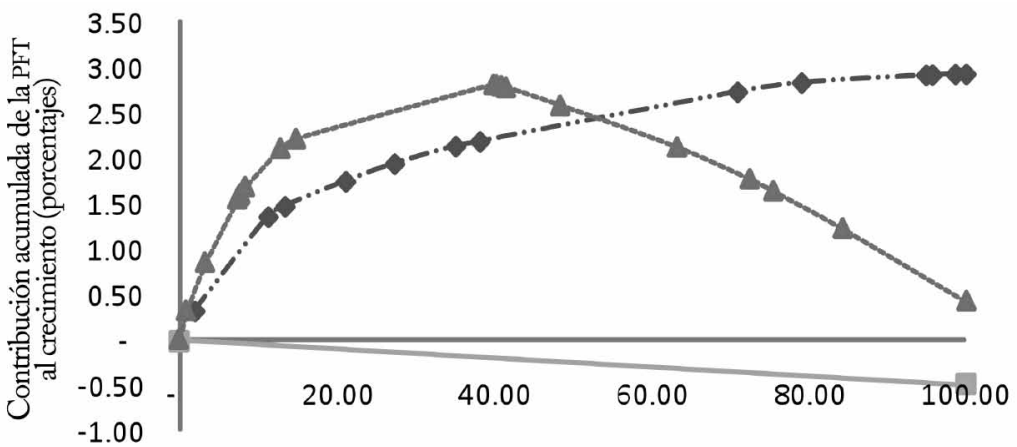

Contribución al valor agregado acumulado (porcentajes)

$-\sim$ Ganadores $\quad-$ Distrito Federal $\rightarrow-$ Resto

Fuente: Elaboración propia con base en información de los censos industriales, de manufacturas o económicos de México (INEGI), según sea el caso. 
si se incluye a los estados con tasas de crecimiento de la PFT negativas, es decir Yucatán, Nuevo León, Colima, Guerrero, Quintana Roo, Nayarit, Oaxaca, Campeche, Hidalgo, Chiapas y Morelos, la tasa de crecimiento acumulada de la PFT cae hasta menos 0.71 por ciento.

En la gráfica vi se ilustra lo ocurrido en el periodo 1998-2003, cuando los estados ganadores presentan una tasa máxima de crecimiento acumulado de $2.93 \%$, mientras que el resto del país tiene una tasa máxima acumulada de $2.82 \%$. Pero, nuevamente, al incluir a los estados con tasas de crecimiento negativas en esta clasificación, la tasa de la PFT observada es de $0.43 \%$. Mientras tanto, el Distrito Federal, el gran perdedor, presenta una tasa de crecimiento de menos 0.49 por ciento.

En conclusión, podemos observar que en el segundo subperiodo la PFT es más alta para los tres grupos mencionados; sin embargo, conviene destacar que esto se debe en parte a una reducción en los factores o fuentes del crecimiento, como se verá a continuación. Es decir, no se anticipa una recuperación sostenible de la productividad.

Con el objeto de analizar la descomposición de fuentes de crecimiento para cada grupo de estados: ganadores, perdedores y resto del país en el periodo 1988-2003, en las gráficas VII, VIII y IX se presenta el desempeño del empleo, el capital y la PFT a partir de sus niveles de 1988 para cada grupo de estados. En la gráfica vir se observa, para el grupo de estados ganadores del periodo 1988-1998, que el mayor contribuyente al crecimiento del producto es el incremento del empleo y la PET tiene una contribución menor. En el periodo 1998-2003 el crecimiento del empleo sigue siendo el mayor contribuyente, pero acompañado de un crecimiento significativo de la PFT y con una aportación ligeramente negativa del capital.

Para el grupo de entidades definidas como resto del pais, durante el periodo 1988-2003, en la gráfica viII se observa una contribución positiva del empleo y de la PFT, aunque en menor proporción que en el grupo de ganadores, y una menor dinámica de la PFT para dichos años. En el grupo de entidades definidas como perdedoras sólo la contribución del empleo es positiva, mientras que las contribuciones de la PFT y el capital son negativas, como se observa en la gráfica Ix.

En esta sección presentamos un breve panorama de la dinámica de la PFT en las manufacturas de las entidades federativas de México en su conjunto y para las agrupaciones de estados en ganadores, perdedores y resto del país. Con ello damos cuenta del crecimiento económico desde una perspectiva novedosa, que ha contribuido a identificar los retos para hacer sostenible el crecimiento. 


\section{Gráfica VII}

Fuentes de crecimiento en las entidades: ganadoras, 1988-2003

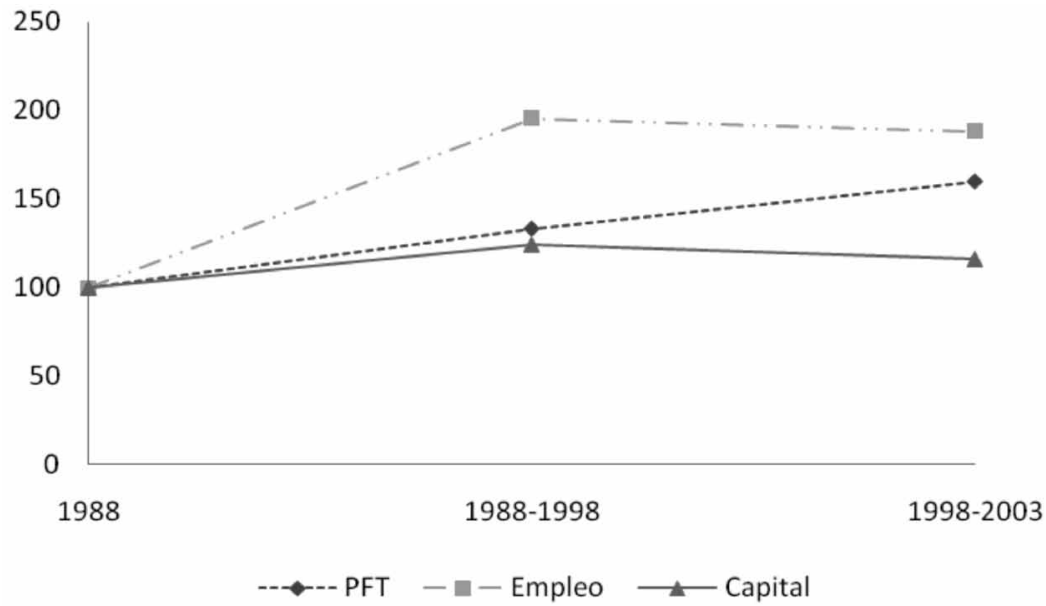

Fuente: Elaboración propia con base en información de los censos industriales, de manufacturas o económicos de México (INEGI), según sea el caso.

\section{Gráfica VIII}

Fuentes de crecimiento en las entidades: resto del país, 1988-2003

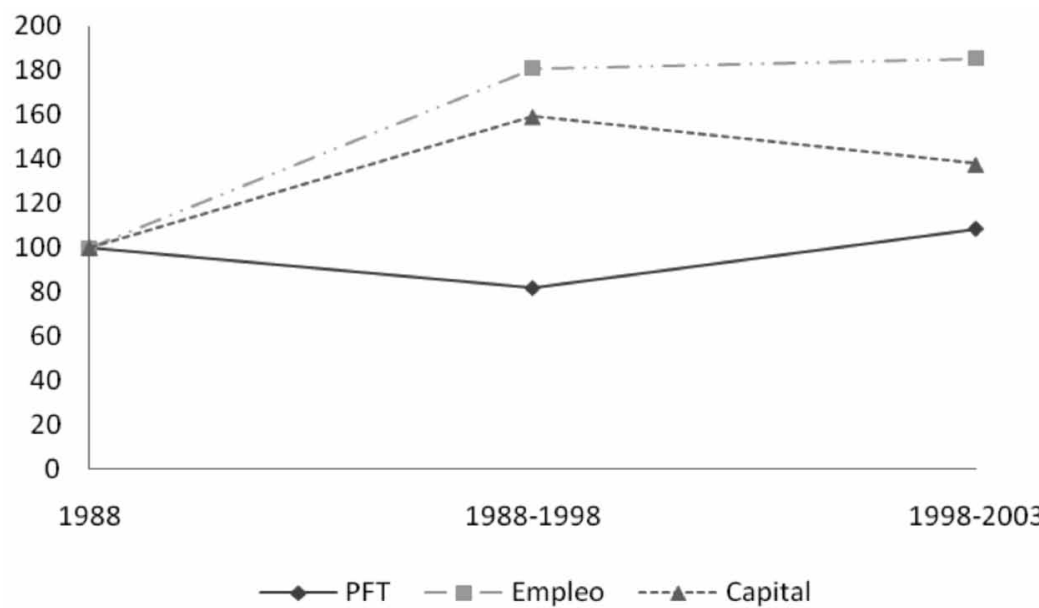

Fuente: Elaboración propia con base en información de los censos industriales, de manufacturas o económicos de México (INEGI), según sea el caso. 


\section{Gráfica IX}

\section{Fuentes del crecimiento en las entidades: perdedoras, 1988-2003}

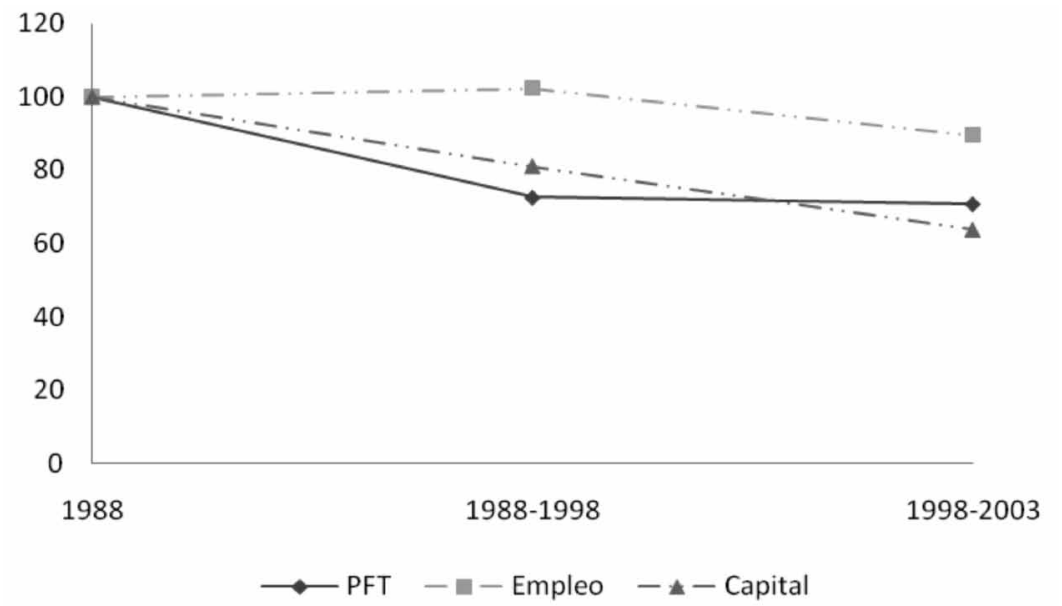

Fuente: Elaboración propia con base en información de los censos industriales, de manufacturas o económicos de México (INEGI), según sea el caso.

\section{Conclusiones y limitaciones}

Tomando en cuenta las limitaciones de la información que se detallan más adelante, aplicando la metodología de contabilidad de crecimiento en las manufacturas estatales de México, se corrobora la evidencia a favor de una muy baja tasa de crecimiento de la productividad factorial en las manufacturas mexicanas para el periodo 1970-2003 (cuadro A.5), aunque es relativamente menos baja para el periodo 1988-2003. Asimismo, se observa que la contribución mayor al crecimiento del producto se explica por un significativo incremento del empleo, y en menor medida de la PFT. Respecto a la contribución del capital, ésta ha sido limitada.

En este artículo se mostró también, a partir de la construcción de los diagramas sunset/sunrise a la manera de Harberger, la expresión espacial de la dinámica del crecimiento económico en las entidades federativas, así como los cambios ocurridos durante el periodo 1988-2003. Con fundamento en la información que sirvió de base a tales diagramas fue posible identificar tres grupos de entidades en función de su desempeño productivo: uno de ganadoras, otro de perdedoras y un tercero conformado por el resto del país. Tales entidades nos permitieron ubicar el patrón espacial de crecimiento en estados de la frontera norte, el centro-occidente y el oriente, mientras que el gran perdedor es el Distrito Federal, lo que sugiere la necesidad de ampliar esta investigación de manera que se profundice en las causas de este desempeño. 
Detrás del análisis de las fuentes de crecimiento en el desempeño de estas regiones están, como en los datos agregados nacionales, la contribución del empleo y la dinámica de la PFT, en ese orden, y destaca que es limitado el papel del capital.

Después de plantear las conclusiones, conviene revisar algunas limitaciones de esta investigación, en particular aquéllas que provienen de las características de la información disponible. Como se mencionó, la información se obtuvo de los censos industriales o de manufactura, que intentan abarcar el universo de todas las empresas manufactureras, y de hecho es la fuente más amplia de que se puede disponer al respecto. Aunque hay diferentes criterios de cobertura, de 1988 en adelante son homogéneos, por lo que las posibles inconsistencias en la cobertura corresponden eventualmente a censos de fechas anteriores a este ańo. Debemos mencionar que a partir de entonces los censos de manufactura se publican como Censos económicos.

Por otra parte, cabe destacar las limitaciones en los índices de precios utilizados, ya que entre ellos se consideró el deflactor implícito del PIB. Esto limita, por ejemplo, la distinción entre bienes exportables y domésticos, pero no se cuenta con un índice de precios con una consistencia similar a nivel estatal para todo el periodo que abarca el estudio. En la medida en que se utilizó como deflactor el deflactor implícito del PIB, generado bajo el Sistema de Cuentas Nacionales, se dispone del mismo para todo el periodo. Para construir la serie se utilizó el correspondiente con base 1980, y posteriormente con base 1993. Conviene hacer notar que se hicieron cálculos utilizando el deflactor implícito de precios de productos manufacturados con resultados similares. No obstante, el uso de este índice nos permite aplicar el mismo deflactor para todas las series nominales, lo que facilita la estimación de la PFT según la recomendación de Harberger (1998).

Otra limitación identificada es la que se relaciona con el tamaño relativo de las economías estatales, ya que un monto significativo de inversión exógena en el estado puede alterar significativamente los cálculos de la PFT y, por tanto, las conclusiones de este tipo de análisis. Otra limitación no menor es el carácter ad hoc de la regionalización. Pero es necesaria para evaluar las implicaciones en términos de productividad del cambio regional en la actividad económica, como se desarrolló en este artículo.

Es relevante señalar que un aspecto que se da como supuesto al identificar la contribución de los factores al crecimiento, es que las remuneraciones de dichos factores reflejan su productividad marginal, pero mientras las condiciones de mercado difieran de aquéllas que suponemos en competencia perfecta, las remuneraciones a los factores productivos tenderán a diferir de sus productividades marginales, por lo que sus con- 
tribuciones al crecimiento también diferirán. En este sentido, con objeto de tener una mejor identificación de las contribuciones factoriales al crecimiento debemos relativizarla en función del grado de competencia existente en sus mercados.

Una limitación que se debe considerar en futuras ampliaciones de esta investigación es la inclusión del papel de los insumos intermedios. Lo anterior en virtud de que el acceso a los insumos, conociendo el tipo de empresa predominante en los estados, como maquiladora/no maquiladora, cabe esperar que tenga diferente trayectoria de costos a lo largo del periodo analizado. Conviene revisar a Amiti y Konings (2005) para conocer el papel de la liberalización comercial en la productividad y la ampliación de la metodología de cálculo de la PFT.

Recientemente, Dreher et al. (2007) atrajeron la atención sobre el papel de la economía informal, o underground economy, en la estimación de la productividad factorial total, dimensión que se debe incluir en los cálculos de productividad para los estados mexicanos, donde el sector informal y la economía subterránea parecen tener una participación relevante y con variaciones estatales.

En cualquier caso, en esta investigación, al contribuir al análisis del crecimiento económico en la economía mexicana a través de la exploración de la dinámica de las manufacturas estatales, se aporta una dimensión no estudiada recientemente que puede apoyar en la identificación de los hechos estilizados del crecimiento y elementos que se deben tomar en cuenta en futuras acciones de política económica regional. 


\section{Anexo}

\section{Cuadro A.1}

Tasas de crecimiento anual promedio de la PFT por estado y contribución de la PFT al crecimiento acumulado, 1970-1988 (\%)

\begin{tabular}{|c|c|c|c|c|c|c|}
\hline Núm. & A. Estado & $\begin{array}{l}\text { B. Tasa de } \\
\text { crecimiento } \\
\text { de la PFT }\end{array}$ & $\begin{array}{l}\text { C. Participa- } \\
\text { ción del valor } \\
\text { agregado }\end{array}$ & $\begin{array}{c}\text { D. Contribu- } \\
\text { ción de la PFT } \\
\text { al crecimiento } \\
\left(B^{*} C\right)\end{array}$ & $\begin{array}{l}\text { E. Participa- } \\
\text { ción del valor } \\
\text { agregado } \\
\text { acumulado }\end{array}$ & $\begin{array}{l}\text { F. Contribu- } \\
\text { ción de la PFT } \\
\text { al crecimiento } \\
\text { acumulado }\end{array}$ \\
\hline 17 & Morelos & 7.11 & 0.81 & 0.06 & 0.81 & 0.06 \\
\hline 20 & Oaxaca & 3.41 & 0.41 & 0.01 & 1.22 & 0.07 \\
\hline 31 & Yucatán & 1.79 & 0.55 & 0.01 & 1.77 & 0.08 \\
\hline 7 & Chiapas & 1.72 & 0.28 & 0.00 & 2.04 & 0.09 \\
\hline 23 & $\begin{array}{l}\text { Quintana } \\
\text { Roo }\end{array}$ & 1.59 & 0.05 & 0.00 & 2.09 & 0.09 \\
\hline 6 & Colima & 1.43 & 0.10 & 0.00 & 2.19 & 0.09 \\
\hline 12 & Guerrero & 1.42 & 0.25 & 0.00 & 2.44 & 0.09 \\
\hline 27 & Tabasco & 1.14 & 0.14 & 0.00 & 2.57 & 0.09 \\
\hline 5 & Coahuila & 0.50 & 3.90 & 0.02 & 6.47 & 0.11 \\
\hline 14 & Jalisco & 0.18 & 5.72 & 0.01 & 12.19 & 0.12 \\
\hline 29 & Tlaxcala & 0.10 & 0.29 & 0.00 & 12.47 & 0.12 \\
\hline 18 & Nayarit & -0.02 & 0.53 & 0.00 & 13.00 & 0.12 \\
\hline 13 & Hidalgo & -0.16 & 1.41 & 0.00 & 14.41 & 0.12 \\
\hline 19 & Nuevo León & -0.30 & 10.85 & -0.03 & 25.26 & 0.09 \\
\hline 11 & Guanajuato & -0.37 & 2.02 & -0.01 & 27.28 & 0.08 \\
\hline 8 & Chihuahua & -0.54 & 1.81 & -0.01 & 29.09 & 0.07 \\
\hline 15 & $\begin{array}{l}\text { Estado de } \\
\text { México }\end{array}$ & -0.68 & 19.53 & -0.13 & 48.61 & -0.06 \\
\hline 25 & Sinaloa & -0.72 & 1.11 & -0.01 & 49.72 & -0.07 \\
\hline 4 & Campeche & -0.76 & 0.24 & 0.00 & 49.97 & -0.07 \\
\hline 9 & $\begin{array}{l}\text { Distrito } \\
\text { Federal }\end{array}$ & -0.77 & 34.99 & -0.27 & 84.96 & -0.34 \\
\hline 28 & Tamaulipas & -0.96 & 1.08 & -0.01 & 86.03 & -0.35 \\
\hline 24 & $\begin{array}{l}\text { San Luis } \\
\text { Potosí }\end{array}$ & -0.99 & 1.13 & -0.01 & 87.17 & -0.36 \\
\hline 2 & $\begin{array}{l}\text { Baja } \\
\text { California }\end{array}$ & -1.01 & 1.85 & -0.02 & 89.01 & -0.38 \\
\hline 3 & $\begin{array}{l}\text { Baja Califor- } \\
\text { nia Sur }\end{array}$ & -2.06 & 0.16 & 0.00 & 89.17 & -0.39 \\
\hline 32 & Zacatecas & -2.14 & 0.10 & 0.00 & 89.27 & -0.39 \\
\hline 21 & Puebla & -2.35 & 3.30 & -0.08 & 92.57 & -0.46 \\
\hline 22 & Querétaro & -2.42 & 0.96 & -0.02 & 93.53 & -0.49 \\
\hline 30 & Veracruz & -2.78 & 3.49 & -0.10 & 97.01 & -0.58 \\
\hline 26 & Sonora & -2.98 & 1.12 & -0.03 & 98.14 & -0.62 \\
\hline 10 & Durango & -3.39 & 0.78 & -0.03 & 98.91 & -0.64 \\
\hline 1 & $\begin{array}{l}\text { Aguasca- } \\
\text { lientes }\end{array}$ & -3.41 & 0.27 & -0.01 & 99.18 & -0.65 \\
\hline 16 & Michoacán & -5.73 & 0.82 & -0.05 & 100.00 & -0.70 \\
\hline
\end{tabular}

Fuente: Elaboración propia con base en la información de los censos industriales, de manufacturas o económicos de México (INEGI), según sea el caso. 


\section{Cuadro A.2}

Tasas de crecimiento anual promedio de la PFT por estado y contribución de la PFT al crecimiento acumulado, 1988-2003 (\%)

\begin{tabular}{|c|c|c|c|c|c|c|}
\hline Núm. & A. Estado & $\begin{array}{l}\text { B. Tasa de } \\
\text { crecimiento } \\
\text { de la PFT }\end{array}$ & $\begin{array}{l}\text { C. Participa- } \\
\text { ción del valor } \\
\text { agregado }\end{array}$ & $\begin{array}{l}\text { D. Contribu- } \\
\text { ción de la PFT } \\
\text { al crecimiento } \\
\left(B^{*} C\right)\end{array}$ & $\begin{array}{l}\text { E. Participa- } \\
\text { ción del valor } \\
\text { agregado } \\
\text { acumulado }\end{array}$ & $\begin{array}{l}\text { F. Contribu- } \\
\text { ción de la PFT } \\
\text { al crecimiento } \\
\text { acumulado }\end{array}$ \\
\hline 27 & Tabasco & 11.79 & 0.71 & 0.08 & 0.71 & 0.08 \\
\hline 16 & Michoacán & 7.21 & 1.19 & 0.09 & 1.90 & 0.17 \\
\hline 7 & Chiapas & 4.92 & 0.53 & 0.03 & 2.44 & 0.20 \\
\hline 8 & Chihuahua & 4.49 & 3.54 & 0.16 & 5.98 & 0.36 \\
\hline 30 & Veracruz & 4.32 & 4.65 & 0.20 & 10.62 & 0.56 \\
\hline 20 & Oaxaca & 3.45 & 1.57 & 0.05 & 12.19 & 0.61 \\
\hline 29 & Tlaxcala & 3.18 & 0.75 & 0.02 & 12.94 & 0.63 \\
\hline 28 & Tamaulipas & 3.12 & 2.50 & 0.08 & 15.44 & 0.71 \\
\hline 21 & Puebla & 3.01 & 3.06 & 0.09 & 18.49 & 0.80 \\
\hline 1 & $\begin{array}{l}\text { Aguasca- } \\
\text { lientes }\end{array}$ & 2.85 & 0.60 & 0.02 & 19.10 & 0.82 \\
\hline 26 & Sonora & 2.45 & 1.81 & 0.04 & 20.91 & 0.87 \\
\hline 6 & Colima & 2.15 & 0.07 & 0.00 & 20.98 & 0.87 \\
\hline 22 & Querétaro & 1.89 & 2.18 & 0.04 & 23.16 & 0.91 \\
\hline 23 & $\begin{array}{l}\text { Quintana } \\
\text { Roo }\end{array}$ & 1.71 & 0.08 & 0.00 & 23.24 & 0.91 \\
\hline 11 & Guanajuato & 1.47 & 4.59 & 0.07 & 27.82 & 0.98 \\
\hline 31 & Yucatán & 1.05 & 0.71 & 0.01 & 28.53 & 0.98 \\
\hline 2 & $\begin{array}{l}\text { Baja } \\
\text { California }\end{array}$ & 0.93 & 1.71 & 0.02 & 30.24 & 1.00 \\
\hline 15 & $\begin{array}{l}\text { Estado de } \\
\text { México }\end{array}$ & 0.90 & 18.17 & 0.16 & 48.41 & 1.16 \\
\hline 10 & Durango & 0.81 & 0.72 & 0.01 & 49.13 & 1.17 \\
\hline 4 & Campeche & 0.76 & 0.08 & 0.00 & 49.21 & 1.17 \\
\hline 14 & Jalisco & 0.39 & 5.87 & 0.02 & 55.08 & 1.19 \\
\hline 32 & Zacatecas & 0.22 & 0.09 & 0.00 & 55.17 & 1.19 \\
\hline 25 & Sinaloa & 0.15 & 0.53 & 0.00 & 55.71 & 1.19 \\
\hline 3 & $\begin{array}{l}\text { Baja Califor- } \\
\text { nia Sur }\end{array}$ & 0.07 & 0.09 & 0.00 & 55.80 & 1.19 \\
\hline 24 & $\begin{array}{l}\text { San Luis } \\
\text { Potosí }\end{array}$ & -0.25 & 2.14 & -0.01 & 57.94 & 1.19 \\
\hline 19 & Nuevo León & -0.26 & 10.06 & -0.03 & 68.00 & 1.16 \\
\hline 5 & Coahuila & -0.38 & 6.40 & -0.02 & 74.40 & 1.14 \\
\hline 13 & Hidalgo & -1.60 & 1.93 & -0.03 & 76.33 & 1.11 \\
\hline 17 & Morelos & -1.61 & 3.17 & -0.05 & 79.50 & 1.06 \\
\hline 9 & $\begin{array}{l}\text { Distrito } \\
\text { Federal }\end{array}$ & -2.17 & 20.04 & -0.43 & 99.54 & 0.62 \\
\hline 12 & Guerrero & -3.45 & 0.20 & -0.01 & 99.74 & 0.61 \\
\hline 18 & Nayarit & -3.52 & 0.26 & -0.01 & 100.00 & 0.60 \\
\hline
\end{tabular}

Fuente: Elaboración propia con base en la información de los censos industriales, de manufacturas o económicos de México (INEGI), según sea el caso. 


\section{Cuadro A.3 \\ Tasas de crecimiento anual promedio de la PFT por estado y contribución de la PFT al crecimiento acumulado, 1988-1998 (\%)}

\begin{tabular}{|c|c|c|c|c|c|c|}
\hline Núm. & A. Estado & $\begin{array}{l}\text { B. Tasa de } \\
\text { crecimiento } \\
\text { de la PFT }\end{array}$ & $\begin{array}{l}\text { C. Participa- } \\
\text { ción del valor } \\
\text { agregado }\end{array}$ & $\begin{array}{l}\text { D. Contribu- } \\
\text { ción de la PFT } \\
\text { al crecimiento } \\
\left(B^{*} C\right)\end{array}$ & $\begin{array}{l}\text { E. Participa- } \\
\text { ción del valor } \\
\text { agregado } \\
\text { acumulado }\end{array}$ & $\begin{array}{l}\text { F. Contribu- } \\
\text { ción de la PFT } \\
\text { al crecimiento } \\
\text { acumulado }\end{array}$ \\
\hline 16 & Michoacán & 10.86 & 1.19 & 0.13 & 1.19 & 0.13 \\
\hline 27 & Tabasco & 9.40 & 0.71 & 0.07 & 1.90 & 0.20 \\
\hline 26 & Sonora & 6.48 & 1.81 & 0.12 & 3.72 & 0.31 \\
\hline 22 & Querétaro & 5.15 & 2.18 & 0.11 & 5.89 & 0.43 \\
\hline 30 & Veracruz & 4.65 & 4.65 & 0.22 & 10.54 & 0.64 \\
\hline 11 & Guanajuato & 3.70 & 4.59 & 0.17 & 15.12 & 0.81 \\
\hline 10 & Durango & 3.54 & 0.72 & 0.03 & 15.85 & 0.84 \\
\hline 1 & $\begin{array}{l}\text { Aguasca- } \\
\text { lientes }\end{array}$ & 3.35 & 0.60 & 0.02 & 16.45 & 0.86 \\
\hline 21 & Puebla & 3.11 & 3.06 & 0.10 & 19.51 & 0.95 \\
\hline 28 & Tamaulipas & 2.83 & 2.50 & 0.07 & 22.01 & 1.02 \\
\hline 5 & Coahuila & 2.14 & 6.40 & 0.14 & 28.41 & 1.16 \\
\hline 29 & Tlaxcala & 1.82 & 0.75 & 0.01 & 29.16 & 1.17 \\
\hline 24 & $\begin{array}{l}\text { San Luis } \\
\text { Potosí }\end{array}$ & 1.19 & 2.14 & 0.03 & 31.30 & 1.20 \\
\hline 8 & Chihuahua & 0.65 & 3.54 & 0.02 & 34.84 & 1.22 \\
\hline 3 & $\begin{array}{l}\text { Baja Califor- } \\
\text { nia Sur }\end{array}$ & 0.52 & 0.09 & 0.00 & 34.93 & 1.22 \\
\hline 15 & $\begin{array}{l}\text { Estado de } \\
\text { México }\end{array}$ & 0.48 & 18.17 & 0.09 & 53.10 & 1.31 \\
\hline 14 & Jalisco & 0.26 & 5.87 & 0.02 & 58.97 & 1.33 \\
\hline 25 & Sinaloa & 0.25 & 0.53 & 0.00 & 59.50 & 1.33 \\
\hline 32 & Zacatecas & 0.13 & 0.09 & 0.00 & 59.59 & 1.33 \\
\hline 2 & $\begin{array}{l}\text { Baja } \\
\text { California }\end{array}$ & 0.03 & 1.71 & 0.00 & 61.30 & 1.33 \\
\hline 31 & Yucatán & -1.21 & 0.71 & -0.01 & 62.01 & 1.32 \\
\hline 19 & Nuevo León & -1.64 & 10.06 & -0.16 & 72.07 & 1.15 \\
\hline 9 & $\begin{array}{l}\text { Distrito } \\
\text { Federal }\end{array}$ & -3.15 & 20.04 & -0.63 & 92.11 & 0.52 \\
\hline 6 & Colima & -3.23 & 0.07 & -0.00 & 92.18 & 0.52 \\
\hline 12 & Guerrero & -3.99 & 0.20 & -0.01 & 92.38 & 0.51 \\
\hline 23 & $\begin{array}{l}\text { Quintana } \\
\text { Roo }\end{array}$ & -4.90 & 0.08 & -0.00 & 92.46 & 0.51 \\
\hline 18 & Nayarit & -5.03 & 0.26 & -0.01 & 92.71 & 0.50 \\
\hline 20 & Oaxaca & -5.38 & 1.57 & -0.08 & 94.28 & 0.41 \\
\hline 4 & Campeche & -5.67 & 0.08 & -0.00 & 94.36 & 0.41 \\
\hline 13 & Hidalgo & -7.11 & 1.93 & -0.14 & 96.30 & 0.27 \\
\hline 7 & Chiapas & -8.45 & 0.53 & -0.05 & 96.83 & 0.23 \\
\hline 17 & Morelos & -11.79 & 3.17 & -0.37 & 100.00 & -0.15 \\
\hline
\end{tabular}

Fuente: Elaboración propia con base en la información de los censos industriales, de manufacturas o económicos de México (INEGI), según sea el caso. 


\section{Cuadro A.4 \\ Tasas de crecimiento anual promedio de la PFT por estado y contribución de la PFT al crecimiento acumulado, 1998-2003 (\%)}

\begin{tabular}{|c|c|c|c|c|c|c|}
\hline Núm. & A. Estado & $\begin{array}{c}\text { B. Tasa de } \\
\text { crecimiento } \\
\text { de la PFT }\end{array}$ & $\begin{array}{l}\text { C. Participa- } \\
\text { ción del valor } \\
\text { agregado }\end{array}$ & $\begin{array}{l}\text { D. Contribu- } \\
\text { ción de la PFT } \\
\text { al crecimiento } \\
\left.\qquad B^{*} C\right)\end{array}$ & $\begin{array}{l}\text { E. Participa- } \\
\text { ción del valor } \\
\text { agregado } \\
\text { acumulado }\end{array}$ & $\begin{array}{l}\text { F. Contribu- } \\
\text { ción de la PFT } \\
\text { al crecimiento } \\
\text { acumulado }\end{array}$ \\
\hline 7 & Chiapas & 35.58 & 0.34 & 0.12 & 0.34 & 0.12 \\
\hline 20 & Oaxaca & 22.05 & 0.92 & 0.20 & 1.26 & 0.32 \\
\hline 17 & Morelos & 17.11 & 1.55 & 0.27 & 2.80 & 0.59 \\
\hline 27 & Tabasco & 16.47 & 1.02 & 0.17 & 3.82 & 0.76 \\
\hline 23 & $\begin{array}{l}\text { Quintana } \\
\text { Roo }\end{array}$ & 14.53 & 0.10 & 0.02 & 3.93 & 0.77 \\
\hline 4 & Campeche & 13.58 & 0.05 & 0.01 & 3.98 & 0.78 \\
\hline 6 & Colima & 13.14 & 0.23 & 0.03 & 4.20 & 0.81 \\
\hline 8 & Chihuahua & 11.13 & 4.82 & 0.54 & 9.02 & 1.34 \\
\hline 13 & Hidalgo & 9.48 & 1.70 & 0.16 & 10.73 & 1.50 \\
\hline 29 & Tlaxcala & 5.33 & 1.10 & 0.06 & 11.83 & 1.56 \\
\hline 31 & Yucatán & 5.26 & 0.75 & 0.04 & 12.58 & 1.60 \\
\hline 30 & Veracruz & 3.56 & 4.00 & 0.14 & 16.58 & 1.75 \\
\hline 28 & Tamaulipas & 3.14 & 3.21 & 0.10 & 19.79 & 1.85 \\
\hline 2 & $\begin{array}{l}\text { Baja } \\
\text { California }\end{array}$ & 2.48 & 4.02 & 0.10 & 23.81 & 1.95 \\
\hline 19 & Nuevo León & 2.39 & 9.49 & 0.23 & 33.30 & 2.17 \\
\hline 1 & $\begin{array}{l}\text { Aguasca- } \\
\text { lientes }\end{array}$ & 1.68 & 1.59 & 0.03 & 34.89 & 2.20 \\
\hline 15 & $\begin{array}{l}\text { Estado de } \\
\text { México }\end{array}$ & 1.66 & 16.94 & 0.28 & 51.84 & 2.48 \\
\hline 21 & Puebla & 1.26 & 4.22 & 0.05 & 56.06 & 2.53 \\
\hline 14 & Jalisco & 0.53 & 8.19 & 0.04 & 64.24 & 2.58 \\
\hline 32 & Zacatecas & 0.42 & 0.42 & 0.00 & 64.66 & 2.58 \\
\hline 16 & Michoacán & 0.21 & 1.51 & 0.00 & 66.17 & 2.58 \\
\hline 25 & Sinaloa & 0.08 & 0.71 & 0.00 & 66.88 & 2.58 \\
\hline 9 & $\begin{array}{l}\text { Distrito } \\
\text { Federal }\end{array}$ & -0.49 & 10.37 & -0.05 & 77.26 & 2.53 \\
\hline 3 & $\begin{array}{l}\text { Baja Califor- } \\
\text { nia Sur }\end{array}$ & -0.61 & 0.13 & -0.00 & 77.39 & 2.53 \\
\hline 18 & Nayarit & -1.96 & 0.24 & -0.00 & 77.62 & 2.53 \\
\hline 12 & Guerrero & -2.79 & 0.23 & -0.01 & 77.86 & 2.52 \\
\hline 24 & $\begin{array}{l}\text { San Luis } \\
\text { Potosí }\end{array}$ & -2.92 & 2.61 & -0.08 & 80.47 & 2.45 \\
\hline 11 & Guanajuato & -3.10 & 5.62 & -0.17 & 86.08 & 2.27 \\
\hline 22 & Querétaro & -3.82 & 3.49 & -0.13 & 89.58 & 2.14 \\
\hline 10 & Durango & -4.40 & 1.13 & -0.05 & 90.71 & 2.09 \\
\hline 26 & Sonora & -4.70 & 3.33 & -0.16 & 94.05 & 1.93 \\
\hline 5 & Coahuila & -5.11 & 5.95 & -0.30 & 100.00 & 1.63 \\
\hline
\end{tabular}

Fuente: Elaboración propia con base en la información de los censos industriales, de manufacturas o económicos de México (INEGI), según sea el caso. 


\section{Cuadro A.5 \\ Tasas de crecimiento anual promedio de la PFT por estado y contribución de la PFT al crecimiento acumulado, 1970-2003 (\%)}

\begin{tabular}{|c|c|c|c|c|c|c|}
\hline Núm. & A. Estado & $\begin{array}{l}\text { B. Tasa de } \\
\text { crecimiento } \\
\text { de la PFT }\end{array}$ & $\begin{array}{l}\text { C. Participa- } \\
\text { ción del valor } \\
\text { agregado }\end{array}$ & $\begin{array}{c}\text { D. Contribu- } \\
\text { ción de la PFT } \\
\text { al crecimiento } \\
\left(B^{*} C\right)\end{array}$ & $\begin{array}{l}\text { E. Participa- } \\
\text { ción del valor } \\
\text { agregado } \\
\text { acumulado }\end{array}$ & $\begin{array}{l}\text { F. Contribu- } \\
\text { ción de la PFT } \\
\text { al crecimiento } \\
\text { acumulado }\end{array}$ \\
\hline 27 & Tabasco & 5.54 & 0.14 & 0.01 & 0.14 & 0.01 \\
\hline 20 & Oaxaca & 3.14 & 0.41 & 0.01 & 0.55 & 0.02 \\
\hline 7 & Chiapas & 3.06 & 0.28 & 0.01 & 0.82 & 0.03 \\
\hline 17 & Morelos & 2.74 & 0.81 & 0.02 & 1.63 & 0.05 \\
\hline 8 & Chihuahua & 2.00 & 1.81 & 0.04 & 3.44 & 0.09 \\
\hline 6 & Colima & 1.93 & 0.10 & 0.00 & 3.54 & 0.09 \\
\hline 23 & $\begin{array}{l}\text { Quintana } \\
\text { Roo }\end{array}$ & 1.70 & 0.05 & 0.00 & 3.58 & 0.09 \\
\hline 29 & Tlaxcala & 1.37 & 0.29 & 0.00 & 3.87 & 0.09 \\
\hline 31 & Yucatán & 1.22 & 0.55 & 0.01 & 4.42 & 0.10 \\
\hline 28 & Tamaulipas & 0.96 & 1.08 & 0.01 & 5.49 & 0.11 \\
\hline 11 & Guanajuato & 0.40 & 2.02 & 0.01 & 7.51 & 0.12 \\
\hline 30 & Veracruz & 0.39 & 3.49 & 0.01 & 11.00 & 0.13 \\
\hline 14 & Jalisco & 0.22 & 5.72 & 0.01 & 16.72 & 0.15 \\
\hline 15 & $\begin{array}{l}\text { Estado de } \\
\text { México }\end{array}$ & -0.02 & 19.53 & -0.00 & 36.24 & 0.14 \\
\hline 5 & Coahuila & -0.02 & 3.90 & 0.00 & 40.14 & 0.14 \\
\hline 21 & Puebla & -0.06 & 3.30 & -0.00 & 43.44 & 0.14 \\
\hline 4 & Campeche & -0.09 & 0.24 & -0.00 & 43.68 & 0.14 \\
\hline 2 & $\begin{array}{l}\text { Baja } \\
\text { California }\end{array}$ & -0.16 & 1.85 & -0.00 & 45.53 & 0.14 \\
\hline 16 & Michoacán & -0.21 & 0.82 & -0.00 & 46.34 & 0.13 \\
\hline 25 & Sinaloa & -0.31 & 1.11 & -0.00 & 47.45 & 0.13 \\
\hline 19 & Nuevo León & -0.31 & 10.85 & -0.03 & 58.31 & 0.10 \\
\hline 26 & Sonora & -0.40 & 1.12 & -0.00 & 59.43 & 0.09 \\
\hline 22 & Querétaro & -0.43 & 0.96 & -0.00 & 60.39 & 0.09 \\
\hline 12 & Guerrero & -0.62 & 0.25 & -0.00 & 60.64 & 0.09 \\
\hline 24 & $\begin{array}{l}\text { San Luis } \\
\text { Potosí }\end{array}$ & -0.65 & 1.13 & -0.01 & 61.77 & 0.08 \\
\hline 1 & $\begin{array}{l}\text { Aguasca- } \\
\text { lientes }\end{array}$ & -0.82 & 0.27 & -0.00 & 62.04 & 0.08 \\
\hline 13 & Hidalgo & -0.87 & 1.41 & -0.01 & 63.45 & 0.06 \\
\hline 3 & $\begin{array}{l}\text { Baja Califor- } \\
\text { nia Sur }\end{array}$ & -1.08 & 0.16 & -0.00 & 63.60 & 0.06 \\
\hline 32 & Zacatecas & -1.10 & 0.10 & -0.00 & 63.71 & 0.06 \\
\hline 9 & $\begin{array}{l}\text { Distrito } \\
\text { Federal }\end{array}$ & -1.36 & 34.99 & -0.48 & 98.70 & -0.41 \\
\hline 10 & Durango & -1.48 & 0.78 & -0.01 & 99.47 & -0.43 \\
\hline 18 & Nayarit & -1.64 & 0.53 & -0.01 & 100.00 & -0.43 \\
\hline
\end{tabular}

Fuente: Elaboración propia con base en la información de los censos industriales, de manufacturas o económicos de México (INEGI), según sea el caso. 


\section{Bibliografía}

Amiti, Mary y Jozef Konings (2005), "Trade Liberalization, Intermediate Inputs and Productivity: Evidence from Indonesia", Working Paper, 05/146, International Monetary Fund <http://www.imf. org/external/pubs/ft/wp/2005/wp05146.pdf>, 9 de septiembre de 2009.

Ascari, Guido y Valeria Di Cosmo (2004), "Determination of Total Factor Productivity in Italian Regions", Working Paper No. 170, Università degli Studi di Pavia <http://economia.unipv.it/docs/ dipeco/quad/ps/q170.pdf>, 11 de septiembre de 2009.

Barro, Robert y Xavier Sala-i-Martin (1995), Economic Growth, McGrawHill, Nueva York.

Boddy, Martin, John Hudson, Anthony Plumridge y Don Webber (2005), "Regional Productivity Differentials: Explaining the Gap", Discussion Papers No. 0515, University of the West of England <http://carecon.org.uk/DP s/0515.pdf>, 24 de agosto de 2009.

Canudas, Rocío del Carmen (2001), "Estudio econométrico de la influencia del capital humano en el crecimiento de la productividad industrial de México, 1960-1993", Estudios Económicos de Desarrollo Internacional, 1 (2), Asociación de Estudios Euro-Americanos de Desarrollo Económico, Santiago de Compostela, pp. 1-16.

Cornberg, Alberto y Francisco Pérez García (eds.) (2010), Fuentes del crecimiento y productividad en Europa y América Latina, Fundación BBVA, Bilbao.

Denison, Edward Fulton (1962), The Sources of Economic Growth in the United States and the Alternatives before Us, New York, Committee for Economic Development, Nueva York.

Díaz-Bautista, Alejandro y Jorge Saénz (2002), "Productividad total factorial y el crecimiento económico en México", Economía y Desarrollo, 1 (1), Universidad Autónoma de Colombia, Bogotá, pp. 105-180.

Dreher, Axel, Pierre-Guillaume Meón y Friedrich Schneider (2007), “The Devil is in the Shadow: Do Institutions Affect Income and Pro- 
ductivity or only Official Income and Official Productivity?", Working Paper No. 2150, CESifo <http://ssrn.com/abstract $=1046641>, 14$ de agosto de 2009 .

Esquivel, Gerardo y Miguel Messmacher (2002), "Sources of Regional (non) Convergence in Mexico", Working Paper, El Colegio de México, México.

Fuentes, Noé, Alejandro Díaz Bautista y Sarah Eva Martínez Pellegrini (coords.) (2003), Crecimiento con convergencia o divergencia en las regiones de México: asimetría centro-periferia, El Colegio de la Frontera Norte-Plaza y Valdés, Tijuana.

Guillermo, Sylvia y Beata Tanka (2007), "Measuring Total Factor Productivity Growth in Mexican Manufacturing: The Story before and after Trade Liberalization”, Ensayos sobre política económica, 25 (53), Banco de la República de Colombia, Bogotá, pp. 168-219.

Harberger, Arnold (1998), "A Vision of the Growth Process", The American Economic Review, 88 (1), American Economic Association, Pittsburgh, pp. 1-32.

Harberger, Arnold (2005), "On the process of the growth and economic policy in developing countries", USAID PPC issued paper 13, University of California, Los Ángeles.

Hanson, Gordon (1994), "Regional Adjustment to Trade Liberalization", Working Paper No. 4713, National Bureau of Economic Research, Cambridge, MA.

Hernández-Laos, Enrique (1984), La productividad y el desarrollo industrial en México, Fondo de Cultura Económica, México.

INEGI (Instituto Nacional de Estadística, Geografía e Informática) (1991), XII Censo Industrial, 1986: datos de 1985, Resumen general, INEGI, México.

INEGI (Instituto Nacional de Estadística, Geografía e Informática) (1991), Censo Económico, 1989: datos de 1988. Resumen general, INEGI, México. 
INEGI (Instituto Nacional de Estadística, Geografía e Informática) (1996), Censo Económico, 1994: datos de 1993. Resumen general, INEGI, México.

INEGI (Instituto Nacional de Estadística, Geografía e Informática) (1999), Censo Económico, 1999: datos de 1998, Resumen general, INEGI, México.

INEGI (Instituto Nacional de Estadística, Geografía e Informática) (2004), Censo Económico, 2004, Resumen general, INEGI, México.

Iregui, Ana María, Luis Melo y María Teresa Ramírez (2006), "Productividad regional y sectorial en Colombia: análisis utilizando datos de panel", Economics Papers, 378, Banco de la República <http://www.banrep.gov.co/docum/ftp/borra378.pdf>, 12 de agosto de 2009.

Jorgenson, Dale Weldeau y Griliches Zvi (1967), “The Explanation of Productivity Change", Review of Economic Studies, 34 (3), Institute for International Economic Studies, Cambridge, MA., pp. 249-280.

Kumar, Surender (2004), "A Decomposition of Total Factor Productivity Growth: A Regional Analysis of Indian Industrial Manufacturing Growth", Working Paper No. 22, National Institute of Public Finance and Policy <http://www.nipfp.org.in/working_paper/ wp04_nipfp_022.pdf>, 15 de agosto de 2009.

León, Adrián de (2000), "Patrones de crecimiento regional y su impacto en la productividad mexicana”, El Mercado de Valores, año 60, 10, Nacional Financiera, México, pp. 35-44.

León, Adrián de (2001), "Recent Regional Changes and Productivity in Mexico", en Christos C. Paraskevopoulos (ed.), The Asymetric Global Economy, Athenian Policy Forum, Toronto, pp. 269-279.

León, Adrián de (2008), “Cambio regional del empleo y productividad manufacturera en México. El caso de la frontera norte y las grandes ciudades: 1970-2004", Frontera Norte, 20 (40), El Colegio de la Frontera Norte, Tijuana, pp. 79-103. 
León, Adrián de (2009), "Crecimiento económico en México: la dinámica de la productividad manufacturera regional, 1970-2003", Ensayos sobre Política Económica, 27 (58), Banco de la República de Colombia, Bogotá, pp. 15-43.

Liu, Bai-Yang y Bong Joon Yoon (2000), "China’s Economic Reform and Regional Productivity Differentials", Journal of Economic Development, 25 (2), The Economic Research Institute, Chung-Ang University, Seúl, pp. 23-41.

Livas-Elizondo, Raúl y Paul Krugman (1992), "Trade Policy and the Third World Metropolis”, Working Paper No. 4238, National Bureau of Economic Research, Cambridge, MA.

Lucas, Robert (1988), "On the Mechanics of Economic Development", Journal of Monetary Economics, 22 (1), Elsevier, Boston, pp. 3-42.

Mankiw, Gregory, David Romer y David Weil (1992), "A Contribution to the Empirics of Economic Growth", Quarterly Journal of Economics, 107 (2), Harvard University, Cambridge, MA., pp. 407-437.

Moomaw, Ronald y Martin Williams (1991), "Total Factor Productivity Growth in Manufacturing: Further Evidence from the States", Journal of Regional Science, 31 (1), Wiley-Blackwell, Nueva York, pp. 17-34.

Spp (Secretaría de Programación y Presupuesto) (1983), XI Censo Industrial, 1981: datos de 1980. Resumen general, spp, México.

SIC (Secretaría de Industria y Comercio) (1973), IX Censo Industrial, 1971: datos de 1970. Resumen general, Dirección General de Estadística, México.

Solow, Robert Merton (1957), "Technical Change and the Agreggate Production Function", Review of Economics and Statistics, 39 (3), Harvard University, Cambridge, MA., pp. 312-320.

Torre, Leonardo de la (2000), "Productivity and Profitability in the Mexican Manufacturing Sector, 1984-1994”, Ph. D. Dissertation, UCLA. 
Recibido: 5 de octubre de 2009. Reenviado: 2 de noviembre de 2010. Aceptado: 24 de enero de 2011.

Adrián de León-Arias. Es doctor en economía. Actualmente es director de la División de Gestión Empresarial del cucEA-Universidad de Guadalajara. Su línea de investigación actual es: desarrollo económico regional en temas de productividad y competitividad. Entre sus últimas publicaciones destacan: "Cambio regional del empleo y productividad manufacturera en México. El caso de la frontera norte y las grandes ciudades: 1970-2004”, Frontera Norte, 20 (40), El Colegio de la Frontera Norte, Tijuana, pp. 79-103 (2008); "Crecimiento económico en México: la dinámica de la productividad manufacturera regional, 1970-2003”, Ensayos sobre Política Económica, 27 (58), Banco de la República de Colombia, Bogotá, pp. 15-43 (2009).

Edna Edith Parra-de la Torre. Es maestra en economía. Sus líneas de investigación actual son: desarrollo económico regional y pobreza. 